\title{
CMT2A Harboring Mitofusin 2 Mutation with Optic Nerve Atrophy and Normal Visual Acuity
}

This article was published in the following Dove Press journal:

International Medical Case Reports Journal

\author{
Silvana Guerriero' \\ Francesco D'Oria' \\ Giacomo Rossetti ${ }^{2}$ \\ Rosa Anna Favale' \\ Stefano Zoccolella' \\ Giovanni Alessio (iD)' \\ Vittoria Petruzzella ${ }^{1}{ }^{\prime}$ \\ 'Department of Medical Science, \\ Neuroscience and Sense Organs, \\ University of Bari Aldo Moro, Bari, Italy; \\ ${ }^{2}$ Department of Molecular Biology, \\ University of Geneva, Geneva, \\ Switzerland
}

\begin{abstract}
Charcot-Marie-Tooth (CMT) constitutes a group of heterogeneous hereditary motor and sensor neuropathies. Mutations in mitofusin-2 (MFN2) cause CMT type 2A by altering mitochondrial fusion and trafficking along with the axonal microtubule system. In literature patients presenting with CMT2A are reported as having a subacute onset of optic atrophy associated with central scotoma and color vision defects. We report on the clinical and genetic findings in a 40 years-old Caucasian woman presenting with CMT type $2 \mathrm{~A}$ and MFN2 mutation (c.2258duplT/p.Leu753fs) who presented bilateral progressive optic atrophy with bilateral severe concentric narrowing of the visual field but normal visual acuity and color vision. This is the first report that describes such phenotypical manifestation of an MFN2 mutation suggesting that the molecular mechanisms underlying the mitofusin-2 function alteration at optic nerve need to be investigated further.
\end{abstract}

Keywords: Charcot-Marie-Tooth type 2A, mitofusin2, optic atrophy, mitochondria, visual field

\section{Introduction}

Charcot-Marie-Tooth diseases (CMT) are a group of clinically and genetically heterogeneous rare disorders of the peripheral nervous system with a prevalence of about 1:2500. ${ }^{1}$ Following neuropathological and electrophysiological criteria, CMT is classified into two major subgroups, i.e. demyelinating CMT type 1 (CMT1), with a severe reduction of nerve conduction velocities $(\mathrm{NCV}<38 \mathrm{~m} / \mathrm{s})$, and axonal CMT type 2 (CMT2), characterized by preferential degeneration of the axon, showing amplitude reductions in NCV but only mildly reduced $(>38 \mathrm{~m} / \mathrm{s})$ or even normal. ${ }^{2}$ Mutations in mitofusin-2 (MFN2) are the primary cause of autosomal dominant CMT form, referred also as CMT2A. ${ }^{2,3}$ During the last decades, CMT2A phenotypes expanded to include white matter alterations, optic atrophy, hearing loss, tremor, pyramidal signs, fatal acute onset encephalopathy, diaphragmatic weakness, ${ }^{4}$ glaucoma, or neutropenia ${ }^{2}$ and severe scoliosis. ${ }^{5}$ Repeated clinical observation of these features has led to several specifically designated subtypes of CMT. ${ }^{6}$ Literature data show that optic atrophy in CMT2A has a subacute onset and patients develop central scotoma and color vision defects; visual acuity becomes gradually low and fundus examination shows pale optic disk; over the course of several years most individuals experience recovery of visual acuity, color vision ${ }^{2,7-11}$

We describe, for the first time, a CMT2A patient harboring a frameshift mutation in MFN2 who presented a bilateral progressive optic atrophy, with a normal visual acuity, normal color perception and bilateral severe concentric narrowing of the visual field.
Correspondence: Vittoria Petruzzella Dipartimento di Scienze Mediche di Base, Neuroscienze e, Organi di Senso Università degli Studi Aldo Moro, Piazza G. Cesare, Bari 70124, Italy

Tel +390805448530

Fax +390805448538

Email vittoria.petruzzella@uniba.it 


\section{Materials and Methods}

A 40-year-old woman complaining of muscle wasting and bilateral concentric narrowing of the visual field with optic nerve atrophy of unclear etiology was examined. The patient complained about the history of axonal neuropathy and optic nerve atrophy of unclear etiology that began in adolescence. She had trouble standing on her toes since her childhood. She began to have multiple falls and these symptoms slowly progressed over time and by the age of 18 , she was transitioned in a wheelchair. On admission, neurological examination revealed flaccid tetraparesis, more prominent on lower limbs (involving both proximal and distal muscles), distal severe impairment on both vibration and pinprick with absent deep tendom reflexes at four limbs. Cognition was normal (mini-mental state examination 29/30). Funduscopy showed a pale optic disc, whereas macula, blood vessels, and peripheral retina appeared to be normal. Visual acuity was 20/20, bilaterally and intraocular pressure was $14 \mathrm{mmHg}$. At that time, computerized visual field exam (Zeiss Humphrey field analyzer) showed generalized depression of retinal sensitivity with a peripheral deep scotoma, and a central residue of about 20 degrees in the right eye and 10 degrees in the left eye (Figure 1). These findings remained stable over time. Optical coherence tomography (OCT) of retinal fiber thickness (RNFL) examination (OCT, Cirrus Zeiss) showed a reduction of the thickness of the nerve fiber layer especially in the lower and temporal left quadrants (Figure 2A). Ganglion cells OU analysis confirmed the reduction of the macular ganglion cells especially in the temporal quadrants (Figure 2B). Visual evocated potentials (VEPs) showed a pattern with a slight increase in latency time and normal voltage. Scotopic electroretinogram (ERG) showed normal voltages in RE, mild reduction in LE; photopic ERG showed mild reduction in both eyes. Ishiara color plate testing scored normal value. Retinal angiography did not show vascular alteration. Risk factors for optic neuropathy such as smoking, alcohol abuse, and other social and personal habits were excluded. Serum blood tests (including glucose level, vitamin B12, and folate levels, immunoglobulin, autoantibodies, paraneoplastic markers, renal and liver function tests, thyroid function) and heart functions were normal. An electrophysiological assessment with electromyography (EMG) and motor and sensory nerve conduction studies was performed at the four limbs and documented a severe axonal sensory neuropathy. Electrophysiologically, the sensory action potentials could not be recorded in both the median and peroneal nerves, while the motor nerve studies documented severely decreased amplitude of motor action potential, more prominent distally and in the peroneal nerves. Electromyography revealed a pattern of chronic denervation without fibrillation or fasciculation in the four limbs muscles, more severe distally and at lower limbs. The sural nerve biopsy confirmed the electrophysiological findings, revealing a severe loss of myelinated

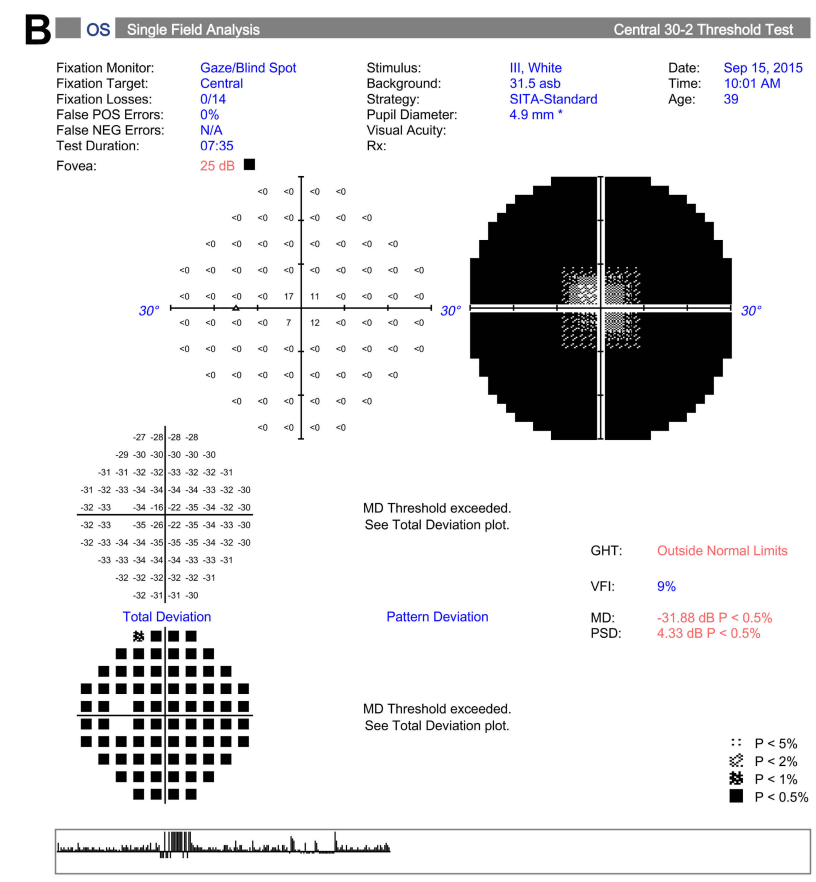

Figure I Visual Field Analysis. Visual field analysis showed peripheral deep scotoma with a central residue of about 20 degrees in the right eye and I0 degrees in the left eye.

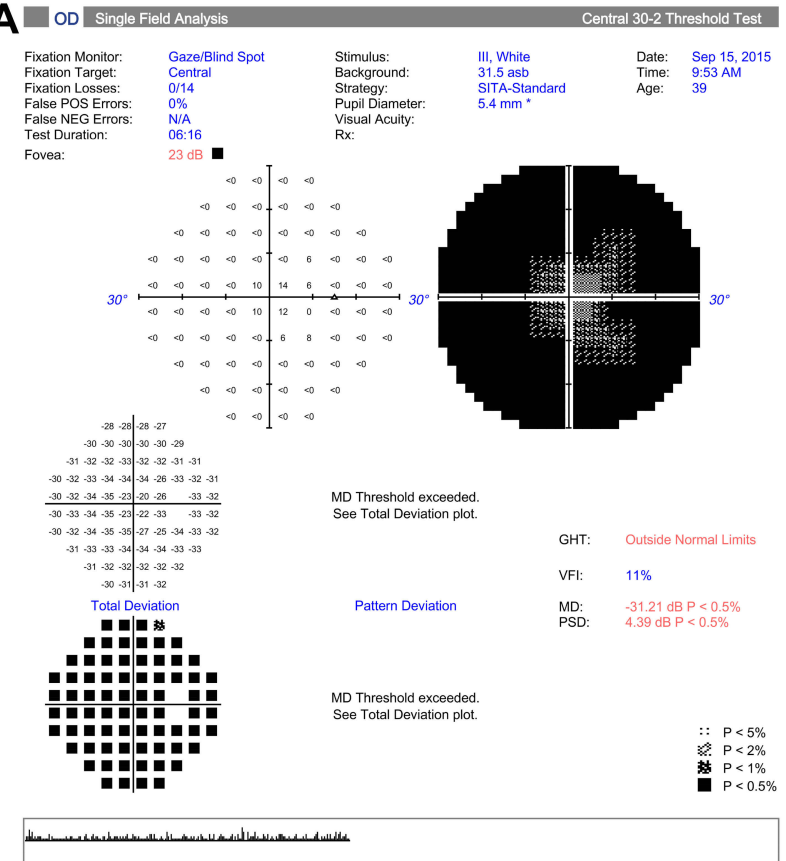



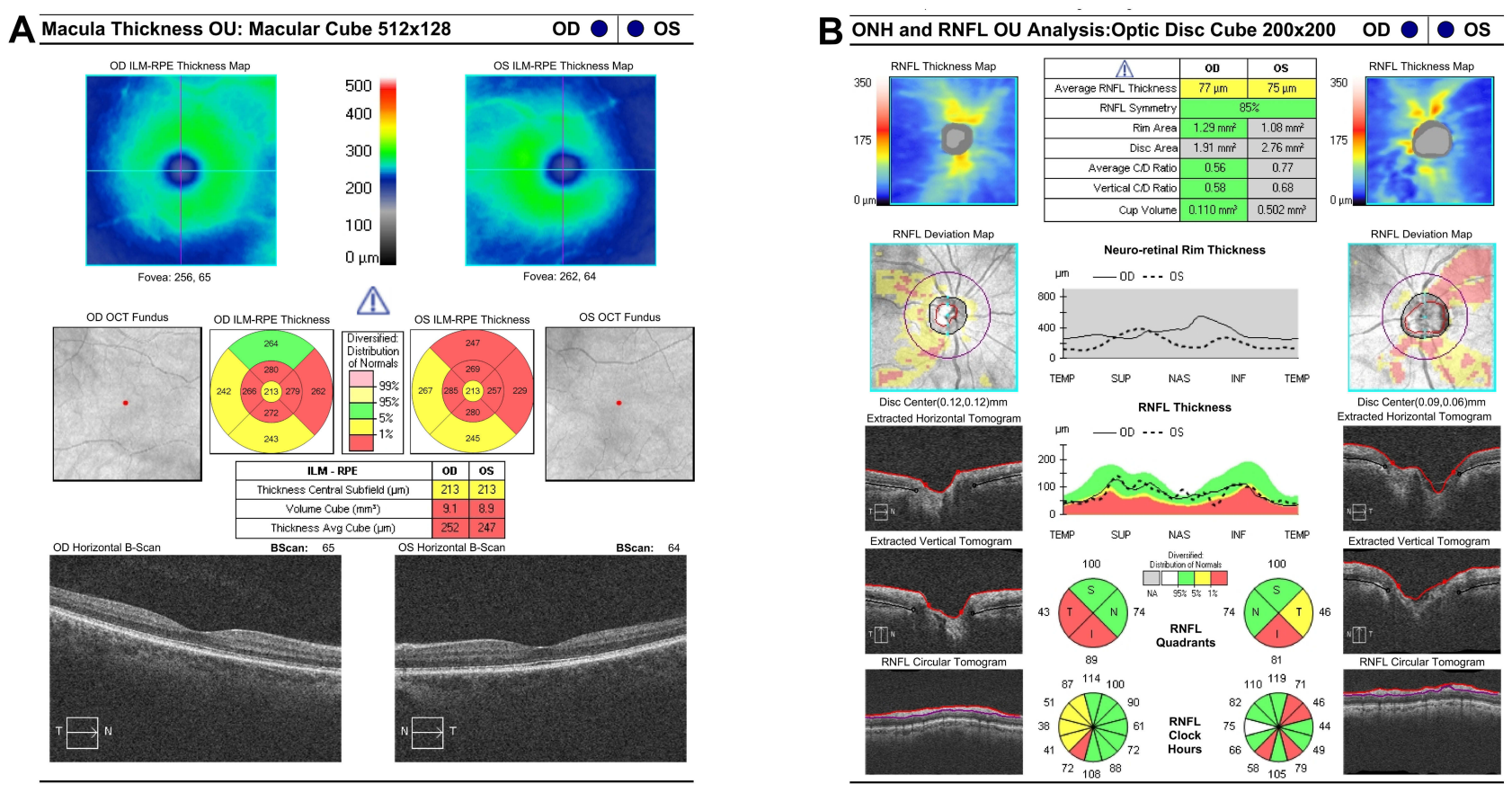

Figure 2 Optical coherence tomography (OCT) exams. (A) OCT of ganglion Cells OU analysis showing reduction of the macular ganglion cells especially in the temporal quadrants. (B) OCT of retinal fiber thickness (RNFL) examination (OCT, Cirrus Zeiss) showing a reduction of the thickness of the nerve fiber layer.

and unmyelinated fibers. Brain Magnetic Resonance (MRI) showed some small subcortical white matter hyperintensities of the vascular hypoxic base with no contrast enhancement. Spectroscopic examination of the basal nuclei showed a reduction of the $\mathrm{N}$-Acetyl-aspartic acid and a moderate increase of choline peak, as for neuronal and axonal suffering. MRI of the spinal cord was normal. The electroencephalogram showed rare high voltage bilateral temporal theta waves. The total body positron emission tomography (PET) examination was normal. 99mTc- (hexamethylpropyle amine oxime) HMPAO scintigraphic examination of brain perfusion showed a little reduction of perfusion of the left occipital lobe. Direct gene testing of the OPA1 gene (MIM 165500) and of the mitochondrial DNA mutations associated with Leber Hereditary Optic Neuropathy (LHON, MIM 53 5000) as well as sequencing of the whole mitochondrial $\mathrm{DNA}^{12}$ in blood were all normal. The screening for MFN2 mutation was performed resulting in the finding of a frameshift mutation c.2258duplT (p. Leu753fs) in exon 19.

\section{Discussion}

Monoallelic pathogenic variants in the MFN2 gene cause two overlapping phenotypes: CMT2A and an autosomal dominant optic atrophy disease. Mutated forms of MFN2 are all known to cause human disease with phenotypes such as encephalopathy, ${ }^{2}$ peripheral neuropathy, ${ }^{6}$ and optic atrophy. ${ }^{2,9-11}$ MFN2 is essential for the transport of mitochondria along axons such as for several other intracellular pathways (i.e. cell cycle progression, maintenance of mitochondrial bioenergetics, apoptosis, and autophagy). In the presence of MFN2 mutations, mitochondrial dynamics and mobility are reduced ${ }^{7}$ thus affecting intra/intercellular distribution and holding a key role in mitochondrial axonal transport, an important process for the extended neuronal axons, especially peripheral nerves. To date, more than 100 pathogenetic mutations in MFN2 have been described, resulting in the different neurological clinical spectrum, ranging from hereditary neuropathies to more severe clinical phenotypes. Pathogenic variants in MFN2 mainly manifest as dominant trait, though in a few sporadic or familial cases, also biallelic or homozygous mutations have been reported. ${ }^{13}$ Recently, an adult patient with pure sensory and autonomic neuropathy sharing typical symptoms with lateonset hereditary sensory autonomic neuropathy (HSAN), further expanded the spectrum of MFN2-related phenotypes, ${ }^{14}$ making it difficult a genotype-phenotype correlation since the same variant can be associated to varying ages of onset of the disease and can cause different clinical phenotypes even within the same family. ${ }^{15}$

So far, several cases of optic atrophy associated with CMT2A have been reported in the literature: Züchner et al $(2006)^{2}$ described six CMT 2A families with a subacute 
onset of optic atrophy and the subsequent slow recovery of visual acuity in $60 \%$ of the patients. Rouzier et al $(2012)^{9}$ reported a large family with optic atrophy with onset in early childhood resembling the autosomal dominant optic atrophy 'plus' phenotype linked to OPA1 mutations, associated with axonal neuropathy and mitochondrial myopathy in adult life. Bombelli et al $(2014)^{10}$ reported three more cases of CMT2A and optic atrophy, vocal cord palsy, and auditory impairment. Leonardi et al (2015) ${ }^{11}$ described a case of a 40 years old Caucasian male with CMT2A and a heterozygous c.775C [T (p. Arg259Cys)] mutation in MFN2 with an acute-onset optic neuropathy: the patient complained visual deterioration over the entire visual field with a rapid progression within a few hours without significant ocular pain. Ando et al $(2017)^{6}$ described a large case study on 1334 unrelated Japanese patients/families with clinically suspected CMT and reported that four patients with MFN2 mutations showed CMT2A with optic atrophy. El Hattab et al $(2018)^{8}$ reported MFN2related autosomal dominant optic atrophy in 12 individuals from a three-generation family. Interestingly, in all the described reports, contrary to our case, MFN2-related optic atrophy typically started during early childhood and presented as vision impairment, central scotomas, and color vision deficiency. Whereas in the case herein described, a co-occurrence of chronic onset optic neuropathy, with normal visual acuity, no impaired color vision, but severe concentric narrowing of the visual field was found. Previously, the same mutation c.2258duplT (p. Leu753fs) in MFN2 identified in our case, was found in a 73-year-old patient with symmetric distal weakness, increased distal paresis of the legs but not optic atrophy. $^{6,11}$ The p.Leu753fs mutation results in five amino acids longer protein at the C-terminus, probably affecting the dimerization of MFN2 which starts the tethering of fusing mitochondria. These findings suggest that MFN2 alteration hampers mitochondrial dynamics and mobility ${ }^{16}$ thus affecting intra/intercellular distribution. ${ }^{17}$ Correct mitochondria functioning as well as spatial and temporal distribution along nerves is fundamental for ATP provision and are crucial for visual activity.

\section{Conclusion}

In conclusion, the present case describes a CMT2 patient manifesting optic atrophy and severe bilateral concentric narrowing of the visual field, further expanding the allelic heterogeneity of mutations in MFN2. Our report showed that the molecular mechanisms related to mitofusin-2 function need to be investigated better to offer further insights into the pathophysiology of optic neuropathy.

\section{Ethics Approval and Consent to Participate and for Publication}

Before recruitment into our study the patient has signed written informed consent for the molecular studies of herself. Research adhered to the tenets of the Declaration of Helsinki. The patient has signed written informed consent for publication of the medical data of herself and she acknowledged that she cannot be identified via the paper; we have fully anonymized it. The Department of Basic Medical Sciences, Neurosciences and Sense organs approved the work for publication.

\section{Acknowledgments}

The authors thank the patient for participating. Funding by 'Ricerca di Ateneo' 2011 and Petruzzella 00724113 Prin 2009 from the University of Bari, Bari, Italy was instrumental in providing support for design, collection and execution of the technical work and for analysis.

\section{Disclosure}

The authors report no conflicts of interest in this work.

\section{References}

1. Skre H. Genetic and clinical aspects of Charcot-Marie-Tooth disease. Clin Genet. 1974;6:98-118.

2. Züchner S, De Jonghe P, Jordanova A, et al. Axonal neuropathy with optic atrophy is caused by mutations in mitofusin 2. Ann Neurol. 2006;59:276-281. doi:10.1002/(ISSN)1531-8249

3. Engelfried K, Vorgerd M, Hagedorn M, et al. Charcot-Marie-Tooth neuropathy type $2 \mathrm{~A}$ : novel mutations in the mitofusin 2 gene (MFN2). BMC Med Genet. 2006;7:53.

4. Tan CA, Rabideau M, Blevins A, et al. Autosomal recessive MFN2-related Charcot-Marie-Tooth disease with diaphragmatic weakness: case report and literature review. Am J Med Genet Part A. 2016;170:1580-1584. doi:10.1002/ajmg.a.37611

5. Stuppia G, Rizzo F, Riboldi G, et al. MFN2-related neuropathies: clinical features, molecular pathogenesis and therapeutic perspectives. $J$ Neurol Sci. 2015;356:7-18. doi:10.1016/j.jns.2015.05.033

6. Ando M, Hashiguchi A, Okamoto Y, et al. Clinical and genetic diversities of Charcot-Marie-Tooth disease with MFN2 mutations in a large case study. J Peripher Nerv Syst. 2017;22:191-199. doi:10.1111/jns.2017.22.issue-3

7. Filadi R, Pendin D, Pizzo P. Mitofusin 2: from functions to disease. Cell Death Dis. 2018;9:330. doi:10.1038/s41419-017-0023-6

8. El-Hattab AW, Suleiman J, Almannai M, et al. Mitochondrial dynamics: biological roles, molecular machinery, and related diseases. Mol Genet Metab. 2018;125:315-321. doi:10.1016/j. ymgme.2018.10.003

9. Rouzier C, Bannwarth S, Chaussenot A, et al. The MFN2 gene is responsible for mitochondrial DNA instability and optic atrophy “plus" phenotype. Brain J Neurol. 2012;135:23-34. doi:10.1093/ brain/awr323 
10. Bombelli F, Stojkovic T, Dubourg O, et al. Charcot-Marie-Tooth disease type 2A: from typical to rare phenotypic and genotypic features. JAMA Neurol. 2014;71:1036-1042. doi:10.1001/jamaneurol.2014.629

11. Leonardi L, Marcotulli C, Storti E, et al. Acute optic neuropathy associated with a novel MFN2 mutation. Neurol. 2015;262:1678-1680. doi:10.1007/s00415-015-7756-x

12. Bianco, A., Bisceglia, L., De Caro, M.F. et al. Leber's hereditary optic neuropathy, intellectual disability and epilepsy presenting with variable penetrance associated to the $\mathrm{m} .3460 \mathrm{G}>\mathrm{A}$ mutation and a heteroplasmic expansion of the microsatellite in MTRNR1 gene case report. BMC Med Genet 19. 2018;129. doi:10.1186/s12881018-0644-3

13. Iapadre G, Morana G, Vari MS, Pinto F, et al. A novel homozygous MFN2 mutation associated with severe and atypical CMT phenotype. Eur J Paediatr Neurol. 2018;22:563-567. doi:10.1016/j.ejpn.2017.12.020
14. Wu R, Fu J, Meng L, et al. Late-onset hereditary sensory and autonomic neuropathy expands the phenotypic spectrum of MFN2-related diseases. Neuropathology. 2018;38:463-467. doi:10.1111/neup.12487

15. Dankwa L, Richardson J, Motley WW, et al. A novel MFN2 mutation causes variable clinical severity in a multi-generational CMT2 family. Neuromuscul Disord. 2019;29:134-137. doi:10.1016/j.nmd. 2018.12.008

16. Devine MJ, Kittler JT. Mitochondria at the neuronal presynapse in health and disease. Nat Rev Neurosci. 2018;19:63-80. doi:10.1038/ nrn.2017.170

17. Chandhok G, Lazarou M, Neumann B. Structure, function, and regulation of mitofusin-2 in health and disease. Biol Rev Camb Philos Soc. 2018;93:933-949. doi:10.1111/brv.12378

\section{Publish your work in this journal}

The International Medical Case Reports Journal is an international, peer-reviewed open-access journal publishing original case reports from all medical specialties. Previously unpublished medical posters are also accepted relating to any area of clinical or preclinical science. Submissions should not normally exceed 2,000 words or 4 published pages including figures, diagrams and references. The manuscript management system is completely online and includes a very quick and fair peer-review system, which is all easy to use. Visit http://www.dovepress.com/testimonials.php to read real quotes from published authors. 